\title{
Nanoheterostructure Metal Oxide Gas Sensors: Opportunities and Challenges
}

\author{
Sheikh A. Akbar ${ }^{1}$, Derek R. Miller ${ }^{1}$, Mohamad A. Al-Hashem ${ }^{1}$, Priyanka Karnati ${ }^{1}$, Janine Walker ${ }^{1}$, \\ Patricia A. Morris ${ }^{1}$ \\ ${ }^{1}$ Department of Material Science and Engineering, The Ohio State University, Columbus, OH 43210, \\ United States \\ Corresponding Author: akbar.1@osu.edu
}

\begin{abstract}
The use of nanoheterostructures as gas sensors has shown the potential to address issues such as gas selectivity or sensitivity in metal-oxide gas sensors although many outstanding challenges remain that inhibit a bottom-up approach to their design. As more complex heterostructures are used, the mechanisms governing their behavior become more ambiguous requiring precise material and electrical characterization techniques to be used. High spatial resolution microscopy has demonstrated the ability to characterize the band structure and defect states in $\mathrm{SnO}_{2}$ nanomaterials revealing the complex nature of mid-gap defect states present. Impedance spectroscopy on single and multi-nanowire $\mathrm{SnO}_{2}$ gas sensors has demonstrated an ability to discriminate between different contributions to sensor response and reveal the underlying mechanisms involved. These techniques are expected to help address outstanding questions to explain the role that defects and heterostructure types have in the sensing process and deconvolute the complex relationships involved to uncover the underlying mechanisms. Additionally, an Open access Database Of Resistive type gas Sensors (ODORS) that is under development is expected to greatly enhance productivity in the field by enabling more informed research studies and presenting new perspectives.
\end{abstract}

Key words: Nanomaterials, Heterostructures, Impedance Spectroscopy, Defects, Synergistic Effects

\section{Metal Oxide Nanostructure Characterization}

Metal oxide nanostructures have been extensively characterized using the high spatial resolution techniques of STEM VEELS [1] and STEM-CL [2] demonstrating the ability to directly characterize the band structure of nanoheterostructures and the complex mid-gap defect states present in $\mathrm{SnO}_{2}$ nanomaterials (Fig. 1).

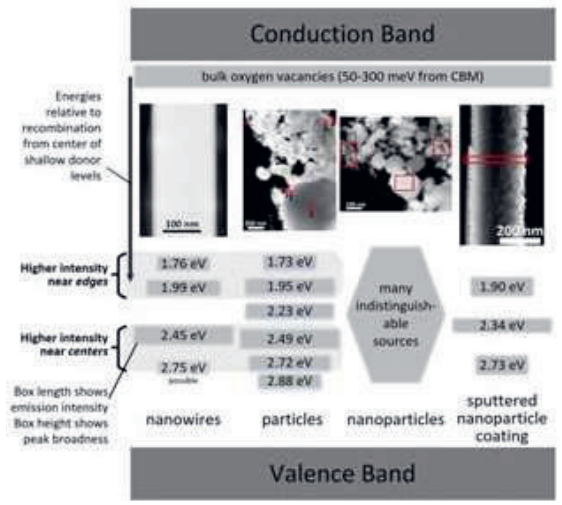

Fig.1.Defect band structure in a few $\mathrm{SnO}_{2}$ nanomaterials [2]. Reprinted with permission.
Given the large number of studies that have been published in the field and the variability in results, a clear understanding of the role and behavior of defects in gas sensing in lacking. To fully understand the role of defects and how they behave under real conditions, careful analysis of the electrical characteristics of these nanostructures under different conditions must be carried out to understand how variables such as annealing temperature, cooling rate or oxygen in/out diffusion affect the behavior of nanostructured metal oxide gas sensors.

\section{Impedance Spectroscopy of Nanostructures}

Impedance measurements carried out on single nanowire (SNW) and multi-nanowire (MNW) $\mathrm{SnO}_{2}$ sensors revealed important information regarding the gas sensing mechanisms of these sensors [3]. Careful circuit analysis revealed that the nanowire junctions in MNW sensors had a greater contribution to the sensor's response compared to the conduction channel modulation while I-V characteristics of the SNW sensors were shown to depend on NW diameter and susceptible to $\mathrm{O}_{2}$ in-diffusion 
processes. Impedance spectroscopy, in conjunction with other techniques, presents a great opportunity to address many outstanding questions in the field of metal oxide gas sensors such as the role of defects in gas sensing, the mechanisms governing the behavior of heterostructures and the complex synergistic effects in multicomponent systems.

\section{Core Shell Nanostructures}

Metal oxide-based gas sensors have many advantages but suffer from poor selectivity and typically require high working temperatures. Nanostructures such as core shell-based gas sensors have been studied to overcome these issues [4]. When designing $n-n, p-n$ or $n-p$ junctions, an equalization of the Fermi level induces the formation of accumulation or depletion layers at the interface through transfer of charges, modifying the conduction channel. Based on the materials used, n-type or p-type behavior have been reported. Parameters such as the thickness of the shell layer, type of sensing material and working temperature have been studied for nanostructure-based gas sensors. Tuning the thickness of the shell layer to within the Debye length of the material has been shown to enhance gas sensing performance [5]. Less attention has been given to understanding how gas sensing measurements are affected by the type of synthesis method and core material used. A better understanding of these parameters is needed to fully grasp the underlying gas sensing mechanisms.

\section{Synergistic Effects}

The complexity of nanostructured metal oxide gas sensor designs increases as the materials, structure design, surface morphologies and other parameters are combined to increase sensitivity and selectivity (Fig. 2). The term "synergistic effects" has been used in literature to describe complimentary behavior between these features. When a gas interacts with a sensor surface, preferential adsorption may be desired to increase selectivity, however if removal of oxygen by surface reactions dictates current modulation then catalytic activity may be more influential than adsorption. A core-shell structure may exhibit better sensitivity due to its capacity for current modulation but not demonstrate selectivity because the surface doesn't promote any preferential reactions or adsorption behavior. Conversely, a mesoporous structure may be used to selectively filter out gasses but have a low response and long recovery time. The interactions between these parameters should be investigated by observing reaction rates, surface adsorption, and structural influence.

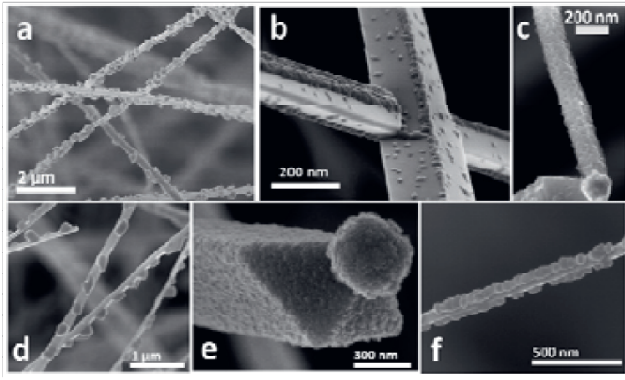

Fig. 2. (a) $\mathrm{Co}_{3} \mathrm{O}_{4}$-decorated $\mathrm{SnO}_{2}$ nanowire by vapor deposition; (b) $\mathrm{Cr}_{2} \mathrm{O}_{3}$-decorated $\mathrm{SnO}_{2}$ nanowires by vapor deposition; (c) $\mathrm{TiO}_{2}-\mathrm{SnO}_{2}$ core-shell heterostructure by magnetron sputtering (MS); (d) $\mathrm{Nb}_{2} \mathrm{O}_{5}-\mathrm{SnO}_{2}$ decoratednanowire heterostructure by MS; (e) core-shell $\mathrm{ZnO}$-coated $\mathrm{SnO}_{2}$ nanowire by $\mathrm{MS}$; and (f) $\mathrm{WO}_{3}$-decorated $\mathrm{SnO}_{2}$ nanowire by $\mathrm{MS}$ [6].

\section{Gas Sensing Database}

We are developing an Open-access Database Of Resistive gas Sensors (ODORS) where published data can be stored that will be a useful asset to the gas sensor community. The database will enable researchers to store data on sensors from their publications into a searchable database. When fully implemented, this will enable the gas sensor researcher to easily determine what the latest studies have been as well as the outstanding challenges.

\section{Acknowledgements}

This project is funded by the National Science Foundation grant no. 160914 .

\section{References}

[1] D. R. Miller, R. E. Williams, S. A. Akbar, P. A. Morris, and D. W. McComb, "Measuring optical properties of individual $\mathrm{SnO} 2$ nanowires via valence electron energy-loss spectroscopy," J. Mater. Res., pp. 1-8, 2017.

[2] D. R. Miller, R. E. Williams, S. A. Akbar, P. A. Morris, and D. W. McComb, "STEMCathodoluminescence of $\mathrm{SnO} 2$ nanowires and powders," Sensors Actuators B Chem., vol. 240, pp. 193-203, 2017.

[3] F. Schipani et al., "Conduction mechanisms in $\mathrm{SnO} 2$ single-nanowire gas sensors: An impedance spectroscopy study," vol. 241, pp. 99108, 2017.

[4] S. Wang, Y. Kang, L. Wang, H. Zhang, Y. Wang, and $Y$. Wang, "Organic/inorganic hybrid sensors: A review," Sensors Actuators, B Chem., vol. 182, pp. 467-481, 2013.

[5] A. Mirzaei, J.-H. Kim, H. W. Kim, and S. S. Kim, "How shell thickness can affect the gas sensing properties of nanostructured materials: Survey of literature," Sensors Actuators B Chem., vol. 258, pp. 270-294, 2017.

[6] D. Miller, "Advancing electronic structure characterization of semiconducting oxide nanoheterostructures for gas sensing." $\mathrm{PhD}$ dissertation, Ohio State University, 2017. 